LBL-28471

Preprint

Submitted to Journal of Mathematical Physics

\title{
A Hamiltonian-Free Description of Single Particle Dynamics for Hopelessly Complex Periodic Systems
}

E. Forest

January 1990

Prepared for the U.S. Department of Energy under Contract Number DE-AC03-76SF00098. 


\title{
A Hamiltonian-Free Description \\ of
}

Single Particle Dynamics for Hopelessly Complex

Periodic Systems ${ }^{1}$

\author{
Étienne Forest \\ Exploratory Studies Group, \\ Accelerator and Fusion Research Division, \\ Lawrence Berkeley Laboratory, \\ 1 Cyclotron Road, Berkeley, Ca 94720
}

PACS numbers: 03.20. $+\mathrm{i}, 02.60 .+\mathrm{y}, 29.20 .-\mathrm{c}, 2.20+\mathrm{b}$.

${ }^{1}$ This work is supported by the U.S. Department of Energy under Contract No. DE-AC03-76SF00098. 


\begin{abstract}
We develop a picture of periodic systems which does not rely on the Hamiltonian of the system but on maps between a finite number of time locations. Moser or Deprit-like normalizations are done directly on the maps thereby avoiding the complex timedependent theory. We redefine linear and nonlinear Floquet variables entirely in terms of maps. This approach relies heavily on the Lie representation of maps introduced by Dragt and Finn. One might say that although we do not use the Hamiltonian in the normalization transformation, we are using Lie operators which are themselves, in some sense, pseudo-Hamiltonians for the maps they represent. Our techniques find application in accelerator dynamics or in any field where the Hamiltonian is periodic but hopelessly complex, such as magnetic field design in stellarators.
\end{abstract}




\section{Introduction}

The study of dynamical systems in traditional branches of classical mechanics uses the Hamiltonian as a starting point. By this we mean that both the numerical as well as the analytical work is done directly on the Hamiltonian of the system. In certain applications, such as dynamics of charged particle beams in accelerators, this approach may lead to a dead end. To illustrate this point, one needs only to compare celestial mechanics to accelerator physics. In accelerator physics, we try to understand the potential behavior of circular machines whose Hamiltonian is a very complex position-dependent function (the timelike variable is actually a length denoted by s). For example, even a small synchrotron radiation ring can consist of several dozens of bending magnets, quadrupoles, sextupoles, orbit correction dipoles and RF-cavities. The necessary inclusion of random errors in the simulation of such a device implies that the Hamiltonian will be a horrible periodic s-dependent function with a number of parameters ranging in the thousands. On the other hand, the problems of celestial mechanics, which might have a higher phase space dimensionality, are parametrized by a relatively small number of variables. In addition, accelerator Hamiltonians are very discontinuous in the time-like variable which adds to the complexity of using a formalism devised for smooth time dependence.

Analytical computations such as the normalization procedures of Deprit and others have emphasized the "flow" (i.e. the Hamiltonian) instead of the study of a one period "map" (i.e. a turn around a circular storage ring). 
Accelerator theorists have tried to adapt these tools to the study of circular machines but they always had to restrict themselves to less than realistic problems. For this reason, a new approach for understanding our systems has been developed in recent years: it emphasizes the computation and analysis of large time maps. We believe that the tools which have been developed (software and theory) can be of use in other fields.

\section{A Bit of History}

This new approach did not develop overnight. In fact, it has its roots deep in the field of accelerator physics. To orient the reader, we will present a subjective (not exhaustive) historical perspective of the use of flow and maps in accelerator theory and simulation. Since pioneering work in accelerator physics has often been obscure, a historical perspective may be viewed as an attempt at a proper recognition of such work.

Originally, the use of maps entered in the design of circular accelerators as paraxial (linear) representation of the ray propagation. The theory was derived from the light optics equivalent and consequently the periodic structure of the systems was not properly exploited. Eventually, Courant and Snyder, ${ }^{[1]}$ in their seminal paper on strong focussing, parametrized the motion around a linear ring in terms of invariant quantities, taking full advantage of the pseudo-harmonic oscillator structure of the motion. In fact, as we will see in this paper, the Lie operator associated to the so-called Courant-Snyder invariant is proportional to the Lie operator of the linear one-turn map. 
On another front, the inclusion of sextupoles in a ring worried a few people in the late fifties. Because computers were not very powerful, Meier, Symon, Laslett and others ${ }^{[2]}$ used simple one dimensional maps to guess at the potential harm caused by nonlinearities in a circular machine. As computers got better, maps disappeared from simulation and were replaced by the so-called kick codes which are in fact second order explicit symplectic integrators. In these codes, each time step of the integrator is derivable from a Hamiltonian. These codes are still the main ingredients of brute force simulations ${ }^{[3]}$.

On the nonlinear theoretical front, accelerator theorists tried to compute various relevant quantities such as frequency shifts (shear terms known as tune shifts in accelerator physics) and distortions of the invariants with the help of canonical perturbation theory. They adapted to accelerator problems the algorithms of Poincaré(-Von Zeippel) ${ }^{[4]}$, Moser-Birkhoff ${ }^{[5]}$ and later Deprit. ${ }^{[6]}$ The inherent complexity of our Hamiltonians leads to great technical difficulties in the application of such algorithms to realistic problems.

Meanwhile the use of maps kept creeping in the linear theory. This probably culminated in a series of paper by $\mathrm{Chao}^{[7]}$ in the late seventies where he computed the equilibrium emmitances and spin polarization in a circular electron ring with the help of a map based theory instead of a flow or Hamiltonian approach. One might ask why accelerator theorists continued to write papers on the evaluation of nonlinear quantities relying entirely on the usual canonical perturbation theory while linear calculations often used the more suited map approach. We venture to suggest that the answer to this question is five words: Lie 
representation of the map. Although it is obvious after a little thought that the quantities obtained by standard canonical perturbation theory are present in a power series expansion of the one-turn map ${ }^{[8]}$, the expansion of the final position and momentum (+spin if you care about it) in terms of their initial components is very different in form from the central object of canonical perturbation theory: the Hamiltonian. Consequently without at least an awareness of the Lie representation, accelerator theorists could not have been expected to re-phrase the nonlinear theory in a way suited for circular machine as they did for the linear case.

At this point enters Alex Dragt. In the seventies Dragt and Finn worked on some version of the Deprit algorithm ${ }^{[9]}$ and applied it to various problems of plasma physics ${ }^{[10,11]}$.

Dragt became involved with accelerator theory and with the help of Douglas they wrote the first version of a code for beam optics (MARYLIE) $^{[12]}$ which parametrizes the Taylor series maps in terms of their Lie generators. In collaboration with the author, a normal form algorithm was first introduced in the code MARYLIE by Dragt, Neri and Healy. Finally, Dragt, in an obscure report, introduced for the first time the concept of phase advance from a map point of view ${ }^{[13]}$. His ideas were not complete, but they planted the right seed in the author's mind. At this point it became clear that the extraction of maps and their subsequent analysis (normalization) provided a powerful approach to numerical and analytic computation in accelerator theory. Remarkably, in 1959 ,Meier and Symon (reference 2) used a Lie representation of the map without knowing it. They were studying a map consisting of a rotation followed by a sextupolar kick. By writing a time dependent pseudo-Hamiltonian which generates the exact same map, they were able 
to compute a canonical transformation to simplify it. Their pseudoHamiltonian was the factorized Lie representation of the map proposed by Dragt and Finn. Meier's and Symon's only error was in not trying to extirpate from the theory and the normalization the bogus time dependence.

Most recently, a software development has increased the numerical power of the map approach enormously. Indeed, the extraction of Taylor series representation of maps from simulation codes can be very tedious. Chao and later the author had to restrict themselves to codes where the individual magnets have a simple representation (thin lenses). At most we could extract $5^{\text {th }}$ degree polynomial maps in $3-d+1 / 2$ phase space ${ }^{[14]}$. Fortunately Martin Berz has created a software package, the Differential Algebra Package, which permits exact automatic differentiation of any quantities integrated on the computer, in particular the position and momentum vector which is being evaluated in our simulation $\operatorname{codes}^{[15]}$. The same tools of Berz permit a user to manipulate the resulting power series map into any type of representation and in particular the factored Lie representation suggested by Dragt and Finn. It suffices to say that all the theoretical concepts discussed in this paper have all been implemented for the power series representation thanks to Berz's package ${ }^{[16]}$.

To set the tone, we will review a few concepts about symplectic maps.

\section{A few words about symplectic maps ${ }^{[17,18,19]}$}

A symplectic map $M$ transforms a differentiable function $f\left(z_{0}\right)$ of the 
initial phase space into another function $(\boldsymbol{M} f)\left(\mathbf{z}_{\mathbf{0}}\right)$, where $\mathbf{z}_{\mathbf{0}}=$ $\left(q_{01}, p_{01}, \ldots, q_{0 N}, p_{0 N}\right)$. We say that $M$ is symplectic if it preserves the Poisson bracket of two functions $f$ and $g$ :

$$
\begin{aligned}
& M[f, g]=[M f, M g] \\
& z_{0}=\left(q_{01}, p_{01}, \ldots, q_{O N}, p_{O N}\right)
\end{aligned}
$$

Now consider the motion generated by a Hamiltonian $\mathrm{K}$ from location $\mathrm{s}_{0}$ to $s$. We know that any function $f\left(z_{0}\right)$ will be transformed at location $s$ into a new function $f_{s}\left(z_{0}\right)$. The two functions are connected by a symplectic map $M\left(s_{0}, s\right)$. Using the properties of Hamilton's equations, one can show that $M\left(s_{0}, s\right)$ obeys the following equation ${ }^{[20]}$ :

$$
\begin{aligned}
\frac{d}{d s} M\left(s_{0}, s\right) & =M\left(s_{0}, s\right):-K\left(z_{0} ; s\right): \\
: f: g & =[f, g] \\
M\left(s_{0}, s_{0}\right) & =E=\text { Identity map }
\end{aligned}
$$

Here we follow Dragt's notation for the Lie operator (equation 2b). From equation (2) we deduce that any map of the form $\mathbf{M}\left(\mathbf{z}_{\mathbf{0}}\right)=\exp \left(: \mathrm{f}\left(\mathbf{z}_{\mathbf{0}}\right):\right)$ is a symplectic map.

Equation (2a) is very similar to Schrödinger's equation for the unitary transformation in quantum mechanics. Here however the resulting map will be symplectic. Notice that the generator :-K( $\left.\mathbf{z}_{0} ; \mathrm{s}\right)$ : depends only on the initial phase space variable $\mathbf{z}_{0}$. It is also easy to show that the differential properties of any Lie operator such as : - K: imply that: 


$$
f_{s}\left(z_{0}\right)=\left(M\left(s_{0}, s\right) f\right)\left(z_{0}\right)=f\left(M\left(s_{0}, s\right) z_{0}\right) \equiv f\left(z_{s}\left(z_{0}\right)\right)
$$

Finally it is worth remembering that symplectic maps act in the reverse order from matrix multiplication when expressed in terms of the initial phase space variables. To show this property, we imagine a two steps process:

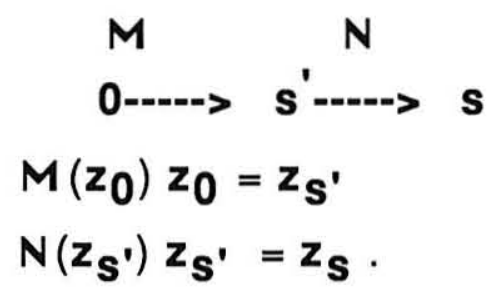

Here the map $M$ transforms functions of the initial phase space at $s=0$ while $\mathbf{N}$ acts on function of the phase space at $\mathbf{s}^{\prime}$ ( thoughout this paper, the notation $\mathbf{M}\left(\mathbf{z}_{\mathbf{0}}\right)$ indicates that the Lie operators of $\mathbf{M}$, are expressed in terms of $\mathbf{z}_{0}$ ).

Clearly, we can propagate a function $f\left(z_{0}\right)$ to a location $s$ by the composition of functions rule:

$$
f_{s}\left(z_{0}\right)=f\left(z_{s}\right)
$$

but,

$$
\begin{aligned}
f\left(z_{\mathbf{s}}\right)= & N\left(z_{s^{\prime}}\right) f\left(z_{s^{\prime}}\right)=N\left(z_{s^{\prime}}\right) M\left(z_{0}\right) f\left(z_{0}\right) \\
& =N\left(M\left(z_{0}\right) z_{0}\right) M\left(z_{0}\right) f\left(z_{0}\right) \\
& =M\left(z_{0}\right) N\left(z_{0}\right) M^{-1}\left(z_{0}\right) M\left(z_{0}\right) f\left(z_{0}\right)
\end{aligned}
$$




$$
=M\left(z_{0}\right) N\left(z_{0}\right) f\left(z_{0}\right)
$$

Equation (5a) is the result of simple composition. In (5b) we apply the definition of the two maps to (5a). Finally, $(5 c)$ and $(5 d)$ are the results of the differential properties of the Lie operators associated to the maps.

Indeed it can be shown using properties (1) and (3) that:

$$
\begin{aligned}
\exp \left(: f\left(\mathbf{M} \mathbf{z}_{\mathbf{0}}\right):\right) & =\exp \left(: \mathbf{M f}\left(\mathbf{z}_{\mathbf{0}}\right):\right) \\
& =\exp \left(\mathbf{M}: f\left(\mathbf{z}_{\mathbf{0}}\right): \mathbf{M}^{-\mathbf{1}}\right)=\mathbf{M} \exp \left(: f\left(\mathbf{z}_{\mathbf{0}}\right):\right) \mathbf{M}^{-\mathbf{1}}
\end{aligned}
$$

The reverse ordering is also present in equation (2a) as seen by integrating it from $s$ to $s+d s$.

We are now ready to introduce our map description of complex periodic systems. In section 2 and appendix 1, we review in very general terms the basic Hamiltonian and canonical transformations used in circular machine theory and simulations. In section $3 a$ and $3 b$, we present the equivalent map description of our system by viewing the ring as an ordered set of maps. In 3c, we sketch the one-turn map normalization. In section 4, we use the one-turn map normalization to define the Floquet ring. In section $\mathbf{5}$, these concepts are applied to the second order normalization of a perturbed Floquet ring. In section 6, we apply the map approach to linear systems in the continuous limit (i.e. in the Hamiltonian limit): a set of well known results follows explicitly. In appendix 2, we sketch the proof of a few theorems. 


\section{The problem of circular machines}

As mentioned in the introduction, the need for a map based theory is most apparent in accelerator physics. Therefore we will described in very general terms the central problem of accelerator design.

Consider a Hamiltonian $\mathrm{H}(\mathbf{x}, \Delta ; \mathrm{s})$ where $\mathbf{x}$ is a phase space vector of dimension $2 \mathrm{~N}$ and $\Delta$ is the set of $\mathrm{N}_{\mathrm{p}}$ parameters describing the departure of our system from its design value (i.e. by definition, the ideal machine is described by the case $\Delta=0$ ). We also assume that $\mathrm{H}$ is periodic in $\mathrm{s}$ with period $s=1$ :

$$
H(x, \Delta ; s+1)=H(x, \Delta ; s) \text {. }
$$

In addition, we can select a new set of canonical variables $\mathbf{z}(\varepsilon)$ which is generated by a periodic Lie operator associated to the function $\mathbf{w}(\mathbf{z}, \Delta ; \mathbf{s} ; \varepsilon)$ :

$$
\begin{aligned}
& \mathrm{d} \mathbf{z} / \mathrm{d} \varepsilon=[\mathbf{z}, \mathrm{w}]=:-\mathrm{w}: \mathbf{z} . \\
& \mathbf{z}(\varepsilon=0)=\mathbf{x}
\end{aligned}
$$

Ultimately the parameter $\varepsilon$ is set to one. It has been shown that the variable $\mathbf{z}(\varepsilon=1)$ is propagated by a Hamiltonian $K(s)$ obeying ${ }^{[21,22]}$ : 


$$
\begin{aligned}
& \mathrm{K}(\mathbf{z}, \Delta ; \mathrm{s})=\mathbf{H}(\mathbf{z}, \Delta ; \mathrm{s})\left(\mathrm{H}(\mathbf{z}, \Delta ; \mathrm{s})+\int_{0}^{\varepsilon=1} \mathrm{~d} \varepsilon \mathbf{A}^{-1}(\mathbf{z}, \Delta ; \mathrm{s}) \frac{\partial}{\partial \mathrm{s}} \mathbf{w}(\mathbf{z}, \Delta ; \mathrm{s} ; \varepsilon)\right), \\
& \frac{\mathrm{d} \mathbf{z}}{\mathrm{ds}}=[\mathbf{z}, \mathrm{K}] .
\end{aligned}
$$

Here $\mathbf{A}^{-1}$ is the periodic canonical map which transforms $\mathbf{x}$ into $\mathbf{z}$ and it is generated by w. Equations (8) and (9) can be viewed as the fundamental equations of an accelerator in the absence of collective or dissipative effects. The fundamental problem of accelerator dynamics is to study the stability of the motion generated by $\mathrm{K}$ or $\mathrm{H}$ as one iterates $n$ turns around the machine from $s=s_{0}$ to $s=s_{0}+n(n-->\infty)$.

Often the theorist attempts to select the generator $w$ in a way that will simplify the structure of $\mathrm{K}$. We refer to this kind of process as a normalization process. In general, the computation of $\mathrm{K}$ is extremely difficult because it requires a knowledge of $\boldsymbol{A}$ for every value of $\mathbf{s}$ ! (For completeness, see appendix 1 for the map equivalent of line (7),(8), and (9) and a derivation of $\mathrm{K}(\mathbf{z}, \Delta ; \mathbf{s})$. )

Typically, no attempt is made to simplify the Hamiltonian and one integrates the motion generated by $\mathrm{H}$ with the help of a symplectic integrator ${ }^{[23]}$. The phase space data is then examined at a finite number of surface of sections (often only one!) and all hope of analytical understanding is abandoned. 
The map description of dynamics described in this paper was developed as a direct consequence of the impossibility to normalize a realistic accelerator Hamiltonian by applying blindly a Deprit type algorithm. 


\section{The Hamiltonian-free or map description}

\section{3.a The motivation}

The map approach is based on a redefinition of the system in terms of a finite number of maps. In accelerator theory, we are motivated in redefining the problem by the following facts:

i) Most simulations are performed by symplectic integrators ${ }^{[24]}$. Only a finite set of location $\left\{s_{j}\right\}$ are examined during this process. The maximum number of locations ever to be examined is the number of integration steps around the ring.

ii) The form of the equations of motion for a computer simulation may not and will not in general be suitable for a normal form analysis.

iii) Although symplectic maps act on the infinite dimensional space of functions, the property displayed in equation (3) permits us to restrict ourselves to the coordinate representation of the map $\mathbf{z}_{\mathbf{s}}\left(\mathbf{z}_{\mathbf{0}}\right)=\mathbf{M} \mathbf{z}_{\mathbf{0}}$.

Statement i) simply states that most simulations involve very discontinuous Hamiltonians in s. While it is cumbersome to fold a discontinuous Hamiltonian into a Deprit style algorithm, the production of maps and their subsequent analysis are ideally suited for discontinuous systems.

Statement ii) points to the necessary discrepancies between the world 
of a computer and the world of theoretical analysis. The process we will describe allows for a total decoupling. One may extract maps using a non-canonical set of coordinates and later transform these maps into canonical variables quite independently of the system which produced these maps. For example, the calculation of the motion through some complex fringe field may be easiest in non-canonical variables: in some cases using even time as the Hamiltonian parameter.

Statement iii) is extremely important. It implies that our efforts should be in the direction of extracting a representation of $\mathbf{z}_{\mathbf{s}}$. For example, in the case of a Taylor series representation of $\mathbf{z}_{\mathbf{s}}$, we mentioned that $M$. Berz has developed powerful software tools (the Differential Algebra Package) which perform automatic differentiation to arbitrary order on a computer ${ }^{[25]}$, making it possible to extract $\mathbf{z}_{\mathbf{s}}$ as a power series around some trajectory in phase space ( usually the periodic closed orbit). The same tools of Berz, allowed J. Irwin and the author to write the necessary software for the normalization of the one-turn map ${ }^{[26]}$. Needless to say that a Taylor series representation may not be always suitable. Presently, non-power series representations are being studied by Warnock et al.They have also developed methods to normalize the map. Their representation can permit the study of very nonlinear processes and they succeed in many cases to find numerically approximate invariant of the motion near chaotic regions ${ }^{[27]}$. Unfortunately, they do not have tools as flexible as the Differential Algebra Package of Berz. For this reason, thanks to Berz's tools, the power series representation of $\mathbf{z}_{\mathbf{s}}$ is the only representation for which all the concepts presented in this paper are and have been implemented. 


\section{3.b The redefinition of the system}

\section{Definition of the ring:}

A ring is an ordered $m$-tuple $\Re=\left(\mathbf{N}_{\mathbf{i}} \mathbf{i}+1\right)$ of $m$ maps connecting $m$ surfaces of sections or observation points. Here the index $\mathbf{i}$ runs from 1 to $m$ with the convention $\mathbf{i}+m=\mathbf{i}$. The maps in $\Re=\left(\mathbf{N}_{\mathbf{i}} \mathbf{i}+1\right)$ are symplectic. Without loss of generality, we assume that these maps transform the origin of phase space into itself:

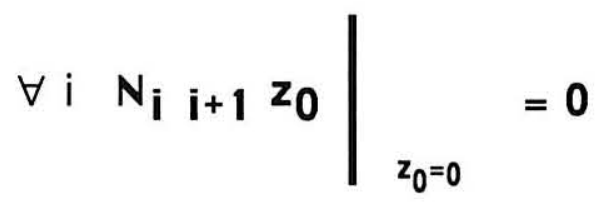

Clearly, the number of maps will depend on the particular aspect of the problem being studied. Again we emphasize that it cannot exceed the number of steps in our symplectic integrator.

We are now in a position to define standard concepts on our ring. The reader must remember that the underlying assumption in this paper is our ability to extract and manipulate maps to any order in the perturbation (i.e. in the case of a power series this would be the degree in the vector $\left(z_{0}, \delta\right)$ where $\delta$ is a subset of the total parameter set $\Delta$ of line 7$)^{[28]}$.

\section{3c. The one-turn maps and their normalization ${ }^{[29]}$}

In the following section we sketch the steps of the normalization of a 
one turn map. This formal procedure is explained in details in reference 16. Given the ring $\Re$, we can easily compute the one-turn map from location $s_{i}$ back to $s_{j}$. This map is simply given by a left to right product:

$$
\begin{aligned}
& \mathbf{M}_{\mathbf{i}}=\prod_{k=i}^{i+m-1} N_{\mathbf{k}} \mathbf{k}+\mathbf{1} \\
& \mathfrak{I}=\left(\mathbf{M}_{\mathbf{i}}\right)
\end{aligned}
$$

The new $m$-tuple $\mathfrak{I}$ which is created out of $\Re$ is not equivalent to $\Re$ as we will see later when we define the Floquet ring.

The first property we will assign to our map is linear stability. To define it

we expand the coordinate representation of $\mathbf{M}_{\mathbf{j}}$ around the origin:

$$
\mathbf{z}_{\mathbf{s}_{\mathbf{i}}}\left(\mathbf{z}_{\mathbf{0}}\right)=\mathrm{Li}_{\mathbf{i}} \mathbf{0}+\ldots
$$

We assume that the $2 \mathrm{~N}$ by $2 \mathrm{~N}$ matrix $\mathrm{L}_{\mathrm{i}}$ has $2 \mathrm{~N}$ distinct eigenvalues on the unit circle:

$$
\lambda_{j}^{ \pm}=\exp \left( \pm \mathrm{i} 2 \pi v_{j}\right), 0<v_{j}<1, j=1, N
$$

Associated to the matrix $L_{i}$ is a Lie map $L_{\mathbf{i}}$. Clearly, the eigenvalues of $L_{i}$ do not depend on location since $\mathbf{L}_{\mathbf{j}}$ is obtained from $\mathbf{L}_{\mathbf{i}}$ by a similarity transformation (the same is true for the full nonlinear map): 


$$
L_{j}=L_{i j}-1 L_{i} L_{i j} \text { where } L_{i j}=\prod_{k=i}^{j-1} L_{k} k+1
$$

For the linear part of the map, we claim that their exist a linear transformation $\mathbf{A}_{\mathbf{L i}}$ such that:

$$
\begin{aligned}
& \mathbf{A}_{\mathbf{L i}} \mathbf{L}_{\mathbf{i}} \mathbf{A}_{\mathbf{L i}}{ }^{-\mathbf{1}}=\exp (:-\mu \cdot \mathbf{J}:)=\mathbf{R}_{\mathbf{L}} \\
& \mu_{j}=2 \pi v_{\mathbf{j}} \\
& J_{j}=\left(\left(z_{0 ; 2 j-1}\right)^{2}+\left(z_{0 ; 2 j}\right)^{2}\right) / 2
\end{aligned}
$$

The transformation $\mathbf{A}_{\mathbf{L i}}$ depends on the location $\mathbf{s}_{\mathbf{j}}$. However the map $\mathbf{R}_{\mathbf{L}}$ is universal, once a tune $v_{\mathrm{j}}$ has been assigned a given plane "j". The transformation done in equation (15) is always possible for a stable linear map with distinct eigenvalues. Let us go back to the nonlinear map $\mathbf{M}_{\mathbf{j}}$. Following Dragt and Finn ${ }^{[30]}$, we express it in a factored form:

$$
\mathbf{M}_{\mathbf{i}}=\mathbf{L}_{\mathbf{i}}\left(\mathbf{L}_{\mathbf{i}}{ }^{-\mathbf{1}} \mathbf{M}_{\mathbf{i}}\right)=\mathbf{L}_{\mathbf{i}} \prod_{k=1}^{N_{0}} \exp \left(: \mathbf{f}_{k}\left(\mathbf{z}_{\mathbf{0}}\right):\right)
$$

In the case of a power series representation of $\mathbf{M}_{\mathbf{i}}, \mathrm{N}_{\mathrm{O}}-1$ is the degree of the polynomial approximation of the function $\mathbf{z}_{\mathbf{s}_{\mathbf{i}}}\left(\mathbf{z}_{\mathbf{0}}\right)$ and $k+2$ is the degree in $\mathbf{z}_{0}$ of the Lie exponent. For a different type of approximation the reader can view $\mathrm{N}_{0}$ and $\mathrm{k}$ as the degree of some smallness parameter. Our ultimate goal, is to normalize $\mathbf{M}_{\mathbf{i}}$ partially or totally. In a total 
normalization, we must find a transformation $\boldsymbol{A}_{\mathbf{i}}$ (analytic around the origin) such that

$$
\mathbf{A}_{\mathbf{i}} \mathbf{M}_{\mathbf{i}} \mathbf{H}_{\mathbf{i}}^{-\mathbf{1}}=\exp (:-\mu \cdot \mathbf{J}+\mathrm{D}(\mathbf{J}):)=\mathbf{R}
$$

For analytic $\mathbf{H}_{\mathbf{j}}$ one can show that $\mathbf{R}$ is unique for a given ordering of the planes and independent of the location (see appendix 2).

Obviously, using equation (15), we factor $\boldsymbol{A}_{\mathbf{i}}$ into a linear and nonlinear part:

$$
\begin{aligned}
& \boldsymbol{A}_{\mathbf{i}}=\boldsymbol{\theta}_{\mathbf{N i}} \boldsymbol{A}_{\mathbf{L i}} \\
& \mathbf{H}_{\mathbf{N i}}=\mathbf{H}_{\mathrm{No}-1 ; \mathbf{i}} \ldots \mathbf{H}_{\mathbf{1} \mathbf{i}} \text {. } \\
& \mathbf{H}_{\mathbf{k i}}=\exp \left(: \mathrm{F}_{\mathrm{k}}:\right) \quad \mathrm{k}=1, \mathrm{~N}_{\mathrm{o}}
\end{aligned}
$$

Applying (18) on $\mathbf{M}_{\mathbf{i}}$ gives us:

$$
\mathbf{A}_{\mathbf{i}} \mathbf{M}_{\mathbf{i}}{\mathbf{\mathbf { A } _ { i }}}^{-\mathbf{1}}=\mathbf{A}_{\mathrm{Ni}} \mathbf{R}_{\mathbf{L}} \prod_{\mathrm{k}=1}^{\mathrm{N}_{\circ}} \exp \left(: \mathrm{g}_{\mathrm{k}}\left(\mathbf{z}_{\mathbf{0}}\right):\right) \mathbf{A}_{\mathrm{Ni}}{ }^{-\mathbf{1}}
$$

where $g_{k} i\left(z_{0}\right)=\boldsymbol{A}_{\mathbf{L i}} f_{k}\left(\mathbf{z}_{\mathbf{O}}\right)=f_{k i}\left(\boldsymbol{\theta}_{\mathbf{L i}} \mathbf{z}_{\mathbf{O}}\right)$.

The normalization of the nonlinear map starts with equation (19). To see the type of operators involved, let us compute the effect of $\boldsymbol{A}_{\mathbf{1}} \mathbf{i}$ on the second order map $\mathbf{R}_{\mathbf{L}} \exp \left(: g_{1} \mathrm{i}:\right.$ :

$$
\begin{aligned}
& \mathbf{A}_{\mathbf{1} \mathbf{i}} \mathbf{R}_{\mathbf{L}} \exp \left(: g_{1} \mathrm{i}:\right) \mathbf{A}_{\mathbf{1 i}}{ }^{-\mathbf{1}}=\exp \left(: \mathrm{F}_{1 \mathrm{i}}:\right) \mathbf{R}_{\mathbf{L}} \exp \left(: g_{1 \mathrm{i}}:\right) \exp \left(:-\mathrm{F}_{1 \mathrm{i}}:\right) \\
& =\mathbf{R}_{\mathbf{L}} \mathbf{R}_{\mathbf{L}}{ }^{-1} \exp \left(: F_{1} ;\right) \mathbf{R}_{\mathbf{L}} \exp \left(: g_{1} i:\right) \exp \left(:-F_{1 j}:\right) \\
& =\mathbf{R}_{\mathbf{L}} \exp \left(: \mathbf{R}_{\mathbf{L}}{ }^{-1} \mathrm{~F}_{1 \mathrm{i}}:\right) \exp \left(: \mathrm{g}_{1 \mathrm{i}}:\right) \exp \left(:-\mathrm{F}_{1 \mathrm{i}}:\right)
\end{aligned}
$$


To first order in the Lie exponents, we can rewrite (20):

$$
\begin{aligned}
& \mathbf{R}_{\mathbf{L}} \exp \left(: \mathbf{R}_{\mathbf{L}}{ }^{-1} \mathrm{~F}_{1} ;:\right) \exp \left(: g_{1} ;:\right) \exp \left(:-\mathrm{F}_{1} ;:\right)
\end{aligned}
$$

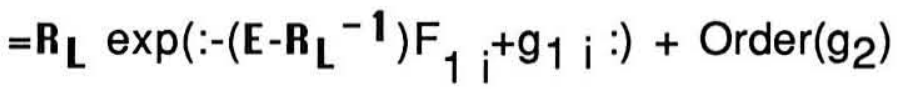

$$
\begin{aligned}
& =\mathbf{R}_{\mathbf{L}} \exp \left(:-\mathrm{TF}_{1 \mathrm{i}}+\mathrm{g}_{1 \mathrm{i}}:\right)+\operatorname{Order}\left(\mathrm{g}_{2}\right) \cdots \\
& \mathbf{T}=\mathbf{E}-\mathbf{R}_{\mathbf{L}}{ }^{-1} \text { where } \mathbf{E} \text { is identity map. }
\end{aligned}
$$

From equation (21), we see that the operator $\mathbf{T}$ is central to the understanding of the effect of any similarity transformation. Since $\mathbf{T}$ is essentially $\mathbf{R}_{\mathbf{L}}$, we must study the Lie operator : $\mu \cdot \mathbf{J}$ :. As pointed out by Cushman,Deprit and Mosak ${ }^{[31]}$ as well as Dragt and Finn ${ }^{[32]}$, the operator $: \mu \cdot \mathrm{J}$ : is a semisimple endomorphism of the space $P_{k}$ of homogeneous polynomials of degree $k \geq 1$ in $z_{0}$; hence it is true that

$$
\mathrm{P}_{\mathrm{k}}=\operatorname{ImT} \oplus \text { KerT }
$$

In fact, it is easy to derive equation (22) by simply constructing the linear eigenfunctions of $\mathbf{T}(\text { or }: \mu \cdot \mathbf{J}:)^{[33]}$.

$$
\begin{gathered}
: \mu \cdot \mathrm{J}: h_{j^{j}}^{ \pm}= \pm i \mu_{j} h_{j}^{ \pm} \\
h_{j}^{ \pm}=z_{2 j-1} \pm i z_{2 j}={\sqrt{2 J_{j}}}_{j} \exp \left(\mp i \varphi_{j}\right) ; \\
{\left[\varphi_{j}, j^{j^{\prime}}\right]=\delta_{j j^{\prime}} \text { being the only nonzero brackets }} \\
J_{j}=\frac{1}{2} h_{j}{ }^{+} h_{j}{ }^{-} \quad j=1, N
\end{gathered}
$$

For completeness, we displayed in (23b) the connection between our 
eigenfunctions and the usual set of action-angle variables $\left(\mathrm{J}_{\mathrm{j}}, \varphi_{\mathrm{j}}\right)$.

Using these linear eigenvectors, we can construct an eigenbasis for $P_{k}$ :

$$
\begin{aligned}
& \mid \mathbf{m}, \mathbf{n}>=\left(h_{1}{ }^{+}\right)^{m_{1}}\left(h_{1}{ }^{-}\right)^{n_{1}} \ldots\left(h_{N^{+}}\right)^{m_{N}}\left(h_{N^{-}}\right)^{n_{N}} \\
& : \mu \cdot J:|m, n>=i(m-n) \cdot \mu| m, n>
\end{aligned}
$$

Since $\mathbf{T}$ is diagonal in the $\mid \mathbf{m}, \mathbf{n}>$ basis, it follows that $\mathrm{P}_{\mathrm{k}}$ decomposes into the direct sum of its image and its kernel. In fact, if the $\mu$ 's are irrational amongst each other, the kernel is given by

$$
\mid \mathbf{m}, \mathbf{n}>\in \operatorname{Ker} \mathbf{T} \Rightarrow \mathbf{m}-\mathbf{n}=\mathbf{0} ; \text { i.e. }|\mathbf{m}-\mathbf{n}|=0 .
$$

In a partial normalization, we decide to leave in the final map terms for which $\mathbf{m}-\mathbf{n} \neq \mathbf{0}$ (or often $(\mathbf{m}-\mathbf{n}) \cdot \mu \cong 0$ ). This allows us to study islands produced by a resonance.

Given a set of positive integers $I_{r}$, we say that $\boldsymbol{M}_{\mathbf{i}}$ is partially normalized into the map $\mathbf{R}$ if :

$$
\begin{aligned}
& \mathbf{A}_{\mathbf{i}} \mathbf{M}_{\mathbf{i}} \mathbf{H}_{\mathbf{i}}^{-\mathbf{1}}=\exp \left(:-\mu \cdot \mathbf{J}+D_{\mathbf{i}}(\mathbf{J})+\sum_{\mathbf{m}-\mathbf{n} \in \mathrm{I}_{r}} D_{\mathbf{m}, \mathbf{n} ; \mathbf{i}} \mid \mathbf{m}, \mathbf{n}>:\right)=\mathbf{R}_{\mathbf{i}} \\
& \text { where } \mathrm{I}_{\mathrm{r}}=\left\{\mathbf{k} \in Z^{N} \mid \mathbf{k}=\text { selected resonances }\right\}
\end{aligned}
$$

As indicated in (26) by the index $\mathrm{i}$, in a partial normalization the final map will depend on the location in the ring. According to equation (21), we obtain $\mathbf{R}$ by inverting $\mathbf{T}$. In fact we can redefine $\mathbf{T}^{-1}$ using a projection operator : 


$$
T_{i m}^{-1} g=T^{-1} P_{i m} g=\sum_{m-n \notin I_{r} \cup\{0\}} \frac{A_{m, n}}{1-\exp (i(m-n) \cdot \mu)} \mid m, n>
$$

$$
\begin{aligned}
& P_{\text {im }} g=\sum_{m-n \notin I_{r} \cup\{0\}} A_{m, n} \mid m, n> \\
& g=\sum_{m, n} A_{m, n} \mid m, n>
\end{aligned}
$$

Provided one knows how to compose the maps involved in the normalization and extract their leading order Lie representation, the maps $\boldsymbol{A}_{\mathbf{k i}}$ can be computed by iteration using $\mathbf{T}_{\mathbf{i} \mathbf{m}}{ }^{-1}$ as defined in equation (27). This procedure was first implemented to $3^{\text {rd }}$ order in the Taylor series by the author and Dragt, in the context of the code MARYLIE . Later Neri and Dragt pushed the process to $5^{\text {th }}$ order using the same code. Recently, the author and Irwin, in close collaboration with Berz, developed the algorithm and software necessary to extend the map normalization to an arbitrary order. The process is semi-analytic since in practice only a small number of components of $\Delta$ (see equations 7,8 , and 9 ) can be retained. This number can vary depending on the order of the normalization, the phase space dimension and the power of the computer used.

For our purpose, it suffices to know that one can define ( exactly for linear maps and formally in the nonlinear case) a normalized map $\mathbf{R}$. Although we concentrate in this article on a normal form algorithm based on the semisimple operator : $\mu \cdot \mathrm{J}$ :, it is possible and sometimes 
desirable to study systems which are not semisimple. What can be done on the Hamiltonian can also be done on the map. 


\section{The Floquet Ring}

Let us assume that we have achieved a complete normalization of the ring. As described by equation (17), we have

$$
\begin{aligned}
& \rho\left(\mathbf{M}_{\mathbf{i}}\right)=\mathbf{A}_{\mathbf{i}} \quad \rho: \mathfrak{I} \rightarrow \text { symplectic maps } \\
& \mathbf{A}_{\mathbf{i}} \mathbf{M}_{\mathbf{i}} \mathbf{A}_{\mathbf{i}}^{-\mathbf{1}}=\exp (:-\mu \cdot \mathbf{J}+\mathrm{D}(\mathbf{J}):)=\mathbf{R}
\end{aligned}
$$

The transformation $\rho$ introduced in (28) can be viewed as a map over the set of one-turn maps $\mathfrak{I}$ defined over $\mathfrak{R}$. Using $\rho$ we can define a new ring: the Floquet ring. We first proceed by mapping the $m$-tuple $\mathfrak{I}$ :

$$
\mathfrak{I}_{\rho}=\rho(\mathfrak{I})=\left(\rho\left(\mathbf{M}_{\mathbf{i}}\right) \mathbf{M}_{\mathbf{i}} \rho\left(\mathbf{M}_{\mathbf{i}}\right)^{-1}\right)=(\mathbf{R}, \mathbf{R} \ldots, \mathbf{R})
$$

The fact that $\mathfrak{I}_{\rho}$ contains only $\mathbf{R}$ is demonstrated in appendix 2 by generalizing equation (14) to the maps $N_{\mathbf{k}} \mathbf{k}+\mathbf{1}$ and using the assumed analyticity of all the maps involved.

More importantly, we must find out what happens to $\mathfrak{R}$. We first state the result.

i) The new ring $\rho(\Re)$ (or $\Re_{\rho}$ ) is made out of amplitude dependent rotations (called phases). The angles of these rotations reduce to the so-called linear phase advances in the linear regime. 
ii) Two different normalization $\rho$ and $\rho$ ' can only differ by a phase for a given ordering of the tunes. Corollary: the phase advance between two matched locations $\left(M_{\mathbf{i}}=\mathbf{M}_{\mathbf{j}}\right)$ is the same for any definition of $\rho$.

\section{PROOF}

We now prove these results.

Property i)

Using the normalization $\rho$, we conclude from equation (29) that :

$$
\rho\left(M_{\mathbf{i}}\right) \mathbf{M}_{\mathbf{i}} \rho\left(\mathbf{M}_{\mathbf{i}}\right)^{-1}=\rho\left(\mathbf{M}_{\mathbf{j}}\right) \mathbf{M}_{\mathbf{j}} \rho\left(\mathbf{M}_{\mathbf{j}}\right)^{-1}=\mathbf{R}
$$

Using the definition of $\mathfrak{I}$, we may write:

$$
M_{j}=N_{i j}{ }^{-1} M_{i} N_{i j}
$$

We can substitute (31) into (30):

$$
\begin{aligned}
& \rho\left(\boldsymbol{M}_{\mathbf{i}}\right) \boldsymbol{M}_{\mathbf{i}} \rho\left(\boldsymbol{M}_{\mathbf{i}}\right)^{-1}=\rho\left(\boldsymbol{M}_{\mathbf{j}}\right) \mathbf{N}_{\mathbf{i j}}^{-1} \boldsymbol{M}_{\mathbf{i}} \boldsymbol{N}_{\mathbf{i j}} \rho\left(\boldsymbol{M}_{\mathbf{j}}\right)-\mathbf{1} \\
& \Rightarrow \quad M_{\mathbf{i}}=\rho\left(M_{\mathbf{i}}\right)^{-1} \rho\left(M_{\mathbf{j}}\right) N_{\mathbf{i j}}^{-1} \boldsymbol{M}_{\mathbf{i}} \mathbf{N}_{\mathbf{i j}} \rho\left(\boldsymbol{M}_{\mathbf{j}}\right)^{-1} \rho\left(M_{\mathbf{i}}\right) \\
& \Rightarrow \quad R=\rho\left(\boldsymbol{M}_{\mathbf{j}}\right) \mathbf{N}_{\mathbf{i j}}{ }^{-1} \rho\left(\boldsymbol{M}_{\mathbf{i}}\right)^{-1} \mathbf{R} \rho\left(\boldsymbol{M}_{\mathbf{i}}\right) \mathbf{N}_{\mathbf{i j}} \rho\left(\mathbf{M}_{\mathbf{j}}\right)^{-1} \\
& \Rightarrow \quad R=\mathbf{B}_{\mathbf{i j}}^{-1} \mathbf{R} \mathbf{B}_{\mathbf{i j}} ; \text { where } \mathbf{B}_{\mathbf{i j}}=\rho\left(\mathbf{M}_{\mathbf{i}}\right) \mathbf{N}_{\mathbf{i j}} \rho\left(\mathbf{M}_{\mathbf{j}}\right)^{-1} \text {. }
\end{aligned}
$$

We now take advantage of the Lie algebraic representation of $\mathbf{R}$ : 


$$
\begin{aligned}
& \mathbf{R}=\mathbf{B}_{\mathbf{i j}}{ }^{-\mathbf{1}} \exp (:-\mu \cdot \mathbf{J}+D(\mathbf{J}):) \mathbf{B}_{\mathbf{i} \mathbf{j}} \\
& \Rightarrow \quad \exp (:-\mu \cdot \mathbf{J}+D(\mathbf{J}):)=\exp \left(: \mathbf{B}_{\mathbf{i j}}{ }^{-\mathbf{1}}(-\mu \cdot \mathbf{J}+D(\mathbf{J})):\right)
\end{aligned}
$$

Using the assumed analyticity of the various maps involved in equation (33), one can show that $\mathbf{B}_{\mathbf{i} \mathbf{j}}$ can depend only on $\mathbf{J}$ (see apendix 2). Hence it can be written with a single Lie operator $\Phi_{\mathrm{ij}}$ :

$$
\mathbf{B}_{\mathbf{i j}}=\exp \left(:-\Phi_{\mathbf{i j}}(\mathbf{J}):\right)
$$

The angle of the rotation produced by $\mathbf{B}_{\mathbf{i j}}$ is simply:

$$
\Delta \Phi_{i j}=-\frac{\partial \Phi_{i j}}{\partial \mathbf{J}}
$$

Using equation (32) and (35), we can write define the Floquet ring $\Re_{\rho}$ associated to $\rho$ to be the $m$-tuple :

$$
\Re_{\rho}=\left(\mathbf{B}_{\mathbf{k}+1}\right) \text { such that } \mathbf{B}_{\mathbf{k} \mathbf{k}+1}=\rho\left(\mathbf{M}_{\mathbf{k}}\right) \mathbf{N}_{\mathbf{k} \mathbf{k}+1} \rho\left(\mathbf{M}_{\mathbf{k}+1}\right)-\mathbf{1}
$$

\section{Property ii)}

Finally, from equation (30) and the uniqueness of $\mathbf{R}$, we obtain a relation identical to equation (33) in the presence of two different normalizations $\rho$ and $\rho^{\prime}$ :

$$
\mathbf{R}=\exp (:-\mu \cdot \mathbf{J}+D(\mathbf{J}):)=\rho\left(\mathbf{M}_{\mathbf{i}}\right) \rho^{\prime}\left(\mathbf{M}_{\mathbf{i}}\right)^{-1} \exp (:-\mu \cdot \mathbf{J}+D(\mathbf{J}):) \rho^{\prime}\left(\mathbf{M}_{\mathbf{i}}\right)^{-1} \rho\left(\mathbf{M}_{\mathbf{i}}\right)
$$


Hence $\rho\left(M_{\mathbf{i}}\right) \rho^{\prime}\left(\mathbf{M}_{\mathbf{i}}\right)^{-1}$ is a rotation and equivalent normalizations can only differ by a phase. It is a simple exercise to prove the corollary on matched locations. 


\section{Perturbation of the Ring $\Re$}

Often one perturbs a Hamiltonian at several locations. One would like to know how the ring $\Re$ and its Floquet counterpart $\rho(\Re)$ are affected by perturbations, in particular Hamiltonian perturbations.

Let us assume that the ring is perturbed at the $\mathrm{i}^{\text {th }}$ location by a Lie operator $\mathbf{C}_{\mathbf{i}}=\exp \left(:-\mathrm{V}_{\mathrm{i}}:\right.$ ). In accelerator physics, this kind of question is often asked. For example, $\mathbf{C}_{\mathbf{i}}$ could represent a nonlinear multipole error or a beam-beam kick: the list is endless. Clearly, the perturbed ring $\Re^{P}$ is just the $m$-tuple:

$$
\Re^{P}=\left(\mathbf{C}_{\mathbf{i}} \mathbf{N}_{\mathbf{i}} \mathbf{i}+\mathbf{1}\right)
$$

More interestingly, we would like to examine the perturbed Floquet ring:

$$
\Re_{\mathbf{f}}^{p}=\left(\mathbf{A}_{\mathbf{i}} \mathbf{C}_{\mathbf{i}} \mathbf{A}_{\mathbf{i}}^{-1} \mathbf{B}_{\mathbf{i} \mathbf{i}+\mathbf{1}}\right)=\left(\exp \left(:-\mathbf{A}_{\mathbf{i}} \mathrm{V}_{\mathbf{i}}:\right) \mathbf{B}_{\mathbf{i}} \mathbf{i}+\mathbf{1}\right)=\left(\exp \left(:-\mathrm{V}_{\mathbf{i}}\left(\mathbf{A}_{\mathbf{i}} \mathbf{z}_{\mathbf{0}}\right):\right) \mathbf{B}_{\mathbf{i}} \mathbf{i}+\mathbf{1}\right)
$$

In his original paper on lattice functions, Dragt refers to $\mathbf{A}_{\mathbf{i}}{ }^{-1}$ as the 'irritability'. In some sense, it gives the true extent of the damage done on the Floquet ring.

As an example of the use of equation (39), let us normalize completly $\Re_{f}^{p}$ to second order in the perturbation $V_{i}$. This has practical application in the design of large synchrotron ring where one needs to keep the shear terms due to sextupoles under control. This process is schematically displayed in figure 2 . 
To proceed as we did before, we first compute the one-turn maps:

$$
\begin{aligned}
& \mathbf{R}_{\mathbf{i}}=\prod_{\mathbf{k}=i}^{i+m-1} \exp \left(:-V_{\mathbf{k}}\left(\mathbf{A}_{\mathbf{k}} \mathbf{z}_{\mathbf{0}}\right):\right) \mathbf{B}_{\mathbf{k}} \mathbf{k}+\mathbf{1} \\
& \mathfrak{I}_{\mathbf{f}}^{\mathrm{p}}=\left(\mathbf{R}_{\mathbf{i}}\right)
\end{aligned}
$$

We then isolate the perturbations on the right side of the factored product of $\mathbf{R}_{\mathbf{i}}$.

$$
\begin{aligned}
& \mathbf{R}_{\mathbf{i}}=\mathbf{R R}^{-\mathbf{1}}\left(\prod_{\mathbf{k}=i}^{i+m-1} \exp \left(:-\mathrm{V}_{\mathbf{k}}\left(\mathbf{B}_{\mathbf{i}} \mathbf{k}^{\mathbf{A}} \mathbf{k}_{\mathbf{z}} \mathbf{\mathbf { z }}\right):\right)\right) \mathbf{R} \\
& \mathbf{R}_{\mathbf{i}}=\mathbf{R}\left(\prod_{\mathbf{k}=i}^{i+m-1} \exp \left(:-\mathrm{V}_{\mathbf{k}}\left(\mathbf{B}_{\mathbf{k}} \mathbf{i}+\boldsymbol{m}^{-\mathbf{1}} \mathbf{A}_{\mathbf{k}} \mathbf{z}_{\mathbf{0}}\right):\right)\right) \\
& \mathbf{R}_{\mathbf{i}}=\mathbf{R} \Pi_{\mathbf{i}}
\end{aligned}
$$


To second order in the perturbation, we can factor $\Pi_{\mathbf{i}}$ :

$$
\begin{aligned}
& \Pi_{\mathbf{i}}=\exp \left(: \mathrm{W}_{1 \mathrm{i}}:\right) \exp \left(: \mathrm{W}_{2 \mathrm{i}}:\right) \ldots \\
& w_{1 i}=\sum_{k=i}^{i+m}-1
\end{aligned}
$$

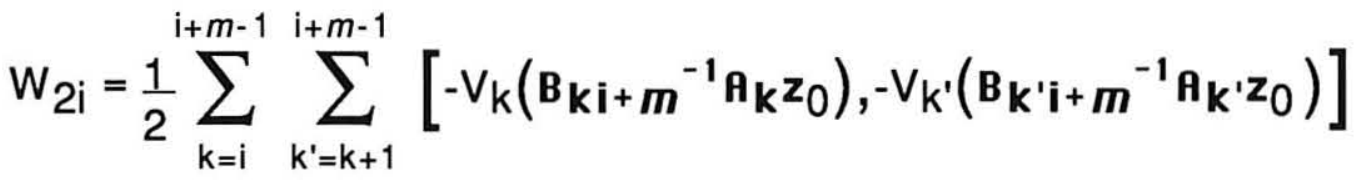

The expression for $\mathrm{W}_{2 \mathrm{i}}$ is the result of a simple application of the Campbell-Baker-Hausdorff formula.

The normalization starts with the application of $\mathbf{A}_{\mathbf{1} \mathbf{i}}=\exp \left(: \mathrm{F}_{1 \mathrm{i}}:\right)$ :

$$
\begin{aligned}
& \mathbf{A}_{1 i} \mathbf{R}_{\mathbf{i}} \mathbf{A}_{1 i}{ }^{-1}=\mathbf{A}_{1 i} \mathbf{R} \Pi_{i} \mathbf{A}_{1 i}{ }^{-1} \\
& F_{1 i}=T_{i m}{ }^{-1} W_{1 i} ; \quad D_{w 1}(J)=\left(E-P_{i m}\right) W_{1 i}
\end{aligned}
$$

In the case of a complete normalization, $D_{w_{1}}(\mathbf{J})$ is often known as the average (or secular) term which in the usual action-angle representation of $(23 b)$ has the form:

$$
D_{w_{1}}(J)=\left\langle W_{1}\right\rangle=\frac{1}{(2 \pi)^{N}} \int_{0}^{2 \pi} \cdots \int_{0}^{2 \pi} W_{1 i}(\varphi, J) d^{N} \varphi
$$


The resulting map is given by:

$$
\begin{aligned}
& \mathbf{A}_{\mathbf{1 i}} \mathbf{R}_{\mathbf{i}} \mathbf{A}_{\mathbf{1 i}}{ }^{-1}=\mathbf{R} \exp \left(: D_{w_{1}}(\mathbf{J}):\right) \exp \left(: \mathrm{W}_{2 \mathrm{i}}^{1}:\right) \\
& \mathrm{W}_{2 \mathrm{i}}^{1}=\frac{1}{2}\left[\mathrm{D}_{\mathrm{w}_{1}}, \mathbf{P}_{\mathbf{i} \mathbf{m}} \mathrm{W}_{1 \mathrm{i}}-2 \mathrm{~F}_{1 \mathrm{i}}\right]+\frac{1}{2}\left[\mathrm{~F}_{1 \mathrm{i}}, \mathbf{P}_{\mathbf{i} \mathbf{m}} \mathrm{W}_{1 \mathrm{i}}\right]+\mathrm{W}_{2 \mathrm{i}}
\end{aligned}
$$

We now proceed with the second order calculation:

$$
\begin{gathered}
F_{2 i}=T_{i m}{ }^{-1} W_{2 i}^{1}=T^{-1} \frac{1}{2}\left[D_{w_{1}}, P_{i m} W_{1 i}-2 F_{1 i}\right] \\
+T_{i m}{ }^{-1}\left(\frac{1}{2}\left[F_{1 i}, P_{i m} W_{1 i}\right]+W_{2 i}\right) \\
D_{w_{2}}(J)=\left(E-P_{i m}\right)\left(\frac{1}{2}\left[F_{1 i}, P_{i m} W_{1 i}\right]+W_{2 i}\right)
\end{gathered}
$$

The first term of $W_{2 i}^{1}$ is entirely in the range of the operator $\mathbf{T}$. This completes the second order normalization process. To second order in the $\mathbf{C}_{\mathbf{i}}$ 's, the Floquet ring is given by:

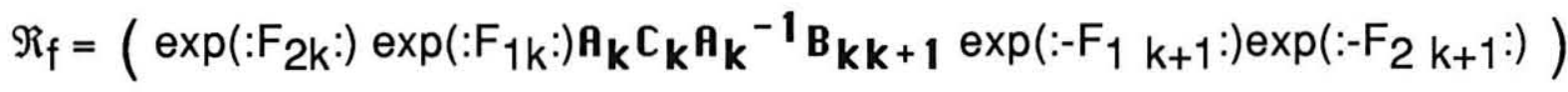

$$
\begin{aligned}
& \mathfrak{I}_{\mathrm{f}}=\left(\mathbf{R} \exp \left(: D_{w_{1}}(\mathbf{J})+D_{w_{2}}(\mathbf{J}):\right)\right)=\left(\exp \left(:-\mu \cdot \mathbf{J}+\mathrm{D}(\mathbf{J})+D_{w_{1}}(\mathbf{J})+D_{w_{2}}(\mathbf{J}):\right)\right)
\end{aligned}
$$




\section{The linear phase advance}

When new techniques are introduced, it is instructive to compare the approach with the old techniques whenever they exist. The difficulty in doing so is proportional to the enhanced power the new methods provide over the old ones. Therefore, while the mathematical equivalence is not in doubt, it is hard to work out a non-trivial and nonlinear example which explicitly displays the mathematical equivalence. Therefore we will settle for a linear example. The reader with a knowledge of accelerator theory will see here an explicit connection between the two methods by letting our ring become an 's-tuple', i.e. by reverting to the Hamiltonian. Clearly, from equation (2a), the Hamiltonian picture corresponds to the maximum ring $\Re_{\infty}$ :

$$
\Re_{\infty}=\lim _{\substack{d s \rightarrow 0 \\ s \in[0,1]}}\left(N_{s} s+d s\right)=\lim _{\substack{d s \rightarrow 0 \\ s \in[0,1]}}\left(E+d s:-H\left(x_{0} ; s\right):\right)
$$

First let us state a few well known results. In the one dimensional case, where the Hamiltonian is given by

$$
H=\frac{1}{2}\left(p^{2}+k(s) q^{2}\right), \quad x=(q, p) .
$$

a stable one-turn map by can be parametrized by the so-called Twiss parameters $^{[34]}$ :

$$
\begin{aligned}
& \mathbf{L}_{\mathbf{s}}=\exp (:-\mu \cdot 1:)=\exp \left(:-\frac{1}{2} \mu \mathrm{c}:\right) \\
& \mathbf{c}=\gamma(\mathbf{s}) q_{0}{ }^{2}+2 \alpha(\mathrm{s}) q_{0} p_{0}+\beta(s) p_{0}^{2} ; \quad 1+\alpha^{2}=\beta \gamma
\end{aligned}
$$


It is easy to verify that the matrix representation $L_{\mathbf{S}}$ of $\mathbf{L}_{\mathbf{s}}$ given by ${ }^{[35]}$ :

$$
L_{S}=\left(\begin{array}{cc}
\cos \mu+\alpha \sin \mu & \beta \sin \mu \\
-\gamma \sin \mu & \cos \mu-\alpha \sin \mu
\end{array}\right)
$$

The invariant c of equation (50) is called the Courant-Snyder invariant in the accelerator physics literature. From our previous discussion is clear that the Twiss parameter $(\alpha, \beta, \gamma)$ are $\mathbf{s}$-dependent. Incidently, the maps $\mathbf{L}_{\mathbf{s}}$ define our one-turn $\infty$-tuple while $\mathrm{H}$ defines the ring:

$$
\begin{aligned}
& \Re_{\infty}=\lim _{\substack{d s \rightarrow 0 \\
s \in[0,1]}}\left(E+d s:-\frac{1}{2}\left(p_{0}{ }^{2}+k(s) q_{0}{ }^{2}\right):\right) \\
& \mathfrak{I}_{\infty}=\left(\mathbf{L}_{s}\right)_{s \in[0,1]} .
\end{aligned}
$$

To proceed further, we must define the map $\rho\left(\mathbf{L}_{\mathbf{s}}\right)$. Following CourantSnyder ${ }^{[36]}$,we define $\rho\left(\mathbf{L}_{\mathbf{s}}\right)$ as :

$$
A_{L_{s}}=\rho\left(L_{s}\right)=\left(\begin{array}{cc}
\sqrt{\beta} & 0 \\
\frac{-\alpha}{\sqrt{\beta}} & \frac{1}{\sqrt{\beta}}
\end{array}\right)
$$

Equation (53) defines uniquely $\boldsymbol{A}_{\mathbf{L} s}$ given a linear map $\mathbf{L}_{\mathbf{s}}$. Given (52) and (53), we will prove using our concepts, that the Floquet ring and the resulting phase advance are given by: 


$$
\begin{gathered}
\Re_{\rho \infty}=\lim _{\substack{d s \rightarrow 0 \\
s \in[0,1]}}\left(E+d s:-\frac{1}{2 \beta}\left(p_{0}^{2}+q_{0}^{2}\right):\right)=\lim _{\substack{d s \rightarrow 0 \\
s \in[0,1]}}\left(E+d s:-\frac{1}{\beta} J:\right) \\
\Delta \Phi_{s_{0} s_{1}}=\int_{s_{0}}^{s_{1}} \frac{d s}{\beta}
\end{gathered}
$$

Before proving a generalization of this result, we point out that the choice of Courant-Snyder was dictated by the kind of perturbation expected in an accelerator. In our machine, we expect the perturbation $\mathbf{C}_{\mathbf{i}}=\exp \left(:-\mathrm{V}_{\mathbf{i}}\right.$ :) of section $\mathbf{5}$ to depend mostly on the position vector $\mathbf{q}$ because the leading contribution to the perturbed Hamiltonian is proportional to the longitudinal component of a magnetic vector potential. Therefore a choice of $\rho\left(L_{S}\right)$ which minimizes the change in the functional form of $V_{i}$ is best. We can generalize the Courant-Snyder choice to a higher dimensionality. The resulting phase advance formula is given for the Hamiltonian

$$
H(\mathbf{x} ; \mathbf{s})=\frac{1}{2} \sum_{i=1, j=1}^{2 N} H_{i j}(s) X_{i} x_{j}
$$

and for $A=\rho(L)$ such that $\quad A_{2 i-12 i-1}>0$ and $A_{2 i-12 i}=0 . i=1, N$ 
by $\{(55 a)$ and $(55 b)\} \Rightarrow \frac{d \Phi_{i}}{d s}=\sum_{j=1}^{N} \frac{H_{2 i j} A_{j 2 i}}{A_{2 i-1} 2 i-1}$

\section{Proof}

We rewrite equation (32) for an infinitesimal change in s:

$$
\begin{aligned}
\mathbf{B}_{\Delta \Phi} & =\exp (:-\Delta \Phi \cdot \mathbf{J})=\boldsymbol{A}_{\mathbf{s}}\left(\mathbf{E}+\mathrm{ds}:-\mathrm{H}\left(\mathbf{x}_{\mathbf{0}} ; \mathbf{s}\right):\right) \mathbf{A}_{\mathbf{s}+\mathbf{d s}}{ }^{-1} \\
\Rightarrow \mathbf{A}_{\mathbf{s}+\mathbf{d s}} & =(\mathbf{E}+: \Delta \Phi \cdot \mathbf{J}:) \mathbf{A}_{\mathbf{s}}\left(\mathbf{E}+\mathrm{ds}:-\mathrm{H}\left(\mathbf{x}_{\mathbf{0}} ; \mathbf{s}\right)\right)+\mathrm{O}\left(\mathrm{ds}^{2}\right) \cdots .
\end{aligned}
$$

Next we assume that $\boldsymbol{A}_{\boldsymbol{s}}$ obeys (55b) and we impose on $\boldsymbol{A}_{\boldsymbol{s}+\mathbf{d s}}$ the same condition ( $\mathrm{j}$ and $\mathrm{k}$ are summed over):

$$
\begin{aligned}
& \boldsymbol{H}_{\mathbf{s}+\mathbf{d s}} \mathrm{X}_{0 ; 2 \mathrm{i}-1}=(\mathbf{E}+: \Delta \Phi \cdot \mathbf{J}:) \boldsymbol{H}_{\mathbf{s}}\left(\mathrm{X}_{0 ; 2 \mathrm{i}-1}+\mathrm{ds}\left[-\mathrm{H}\left(\mathrm{x}_{0} ; \mathrm{s}\right), \mathrm{X}_{0 ; 2 \mathrm{i}-1}\right]\right) \\
& =(\mathbf{E}+: \Delta \Phi \cdot \mathbf{J}:) \boldsymbol{A}_{\mathbf{s}}\left(\mathrm{X}_{0 ; 2 \mathrm{i}-1}+\mathrm{ds} \mathrm{H}_{2 \mathrm{ij}} \mathrm{X}_{0 ; \mathrm{j}}\right) \\
& =(E+: \Delta \Phi \cdot J:)\left(A_{2 i-1 k} X_{0 ; k}+\text { ds } H_{2 i j} A_{j k} X_{0 ; k}\right) \\
& =\left(A_{2 i-1 k} X_{0 ; k}+\left(-\Delta \Phi_{k} A_{2 i-12 k-1} X_{0 ; 2 k}+\Delta \Phi_{k} A_{2 i-12 k} X_{0 ; 2 k-1}+d s H_{2 i j} A_{j k} X_{0 ; k}\right)\right)
\end{aligned}
$$

We extract from (57) the $(2 \mathrm{i}-1,2 \mathrm{i})$ component of $A_{S+d s}$ and set it to zero:

$$
0=d s H_{2 i j} A_{j 2 i}-\Delta \Phi_{i} A_{2 i-12 i-1} \Rightarrow \text { Property (55c). QED }
$$

We can apply this formula to a problem already solved by Edwards and Teng $^{[37]}$ where they accidently chose the same definition for the 
transformation $\rho(L)$.

In their case, the Hamiltonian matrix $\mathrm{H}_{\mathrm{ij}}$ was

$$
H=\left(\begin{array}{cccc}
F & 0 & K & -L \\
0 & 1 & L & 0 \\
K & L & G & 0 \\
-L & 0 & 0 & 1
\end{array}\right)
$$

They parametrized the matrix $A$ as

$$
\begin{aligned}
& A=B C \\
& B=\left(\begin{array}{cc}
I \cos \phi & D^{-1} \sin \phi \\
-D \sin \phi & I \cos \phi
\end{array}\right) ; \cos (\phi)>0, \quad I=\left(\begin{array}{ll}
1 & 0 \\
0 & 1
\end{array}\right), \mathbf{D}=\left(\begin{array}{ll}
a & b \\
c & d
\end{array}\right),|D|=1 \\
& \text { (60b) }
\end{aligned}
$$

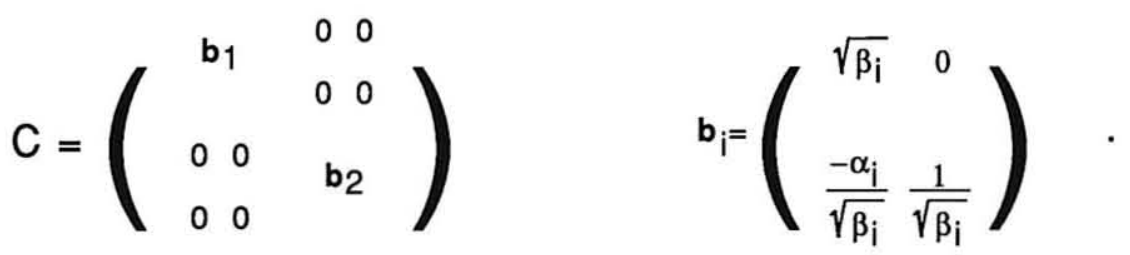

Because the map A obeys (55b), we can compute the phase advance using (55c):

$$
\frac{\mathrm{d} \Phi_{1}}{\mathrm{ds}}=\frac{(1-\mathrm{Lb} \tan (\phi))}{\beta_{1}}
$$




$$
\frac{\mathrm{d} \Phi_{2}}{\mathrm{ds}}=\frac{(1+\mathrm{Lbtan}(\phi))}{\beta_{2}}
$$

These results are exactly those obtained by Teng and Edwards through a totally different method.

In a final exercise, we would like to explore the relationship between two definitions of the phase advance. Going back to the original one dimensional problem of Courant-Snyder, we can examine the following definition:

$$
A_{L_{S}}=\rho^{\prime}\left(L_{S}\right)=\left(\begin{array}{cc}
\frac{1}{\sqrt{\gamma}} & \frac{-\alpha}{\sqrt{\gamma}} \\
0 & \sqrt{\gamma}
\end{array}\right)
$$

If the world was made out of primarily velocity dependent potentials, Courant and Snyder would have selected $\rho$ '. The phase advance is obtained by a symmetry argument (canonically exchanging $q$ and $p$, and applying (55c) ):

$$
\Delta \Phi_{\mathrm{s}_{0} \mathrm{~s}_{1}}=\int_{\mathrm{s}_{0}}^{\mathrm{s}_{1}} \frac{\mathrm{k}(\mathrm{s}) \mathrm{ds}}{\gamma}
$$

Using the ring given by (48) and the underlying Hamiltonian given at line (49), we can derive a famous set of rules for the evolution of the Twiss parameters $(\alpha, \beta, \gamma)$. This will allow us to relate the phase advances of $\rho$ and $\rho^{\prime}$ explicitely. 


$$
\begin{aligned}
& L_{s+d s}=N_{s} s+d s^{-1} L_{s} N_{s} s+d s \\
& =\mathbf{N}_{\mathbf{s}} \mathbf{s}+\mathbf{d s} \mathbf{s}^{-1} \exp \left(:-\frac{1}{2} \mu c:\right) \mathbf{N}_{\mathbf{s}} \mathbf{s}+\mathbf{d s}=\exp \left(:-\frac{1}{2} \mu \mathbf{N}_{\mathbf{s}} \mathbf{s}+\mathbf{d s} \mathbf{s}^{-1} \mathrm{c}:\right) \\
& =\exp \left(:-\frac{1}{2} \mu\left(E-d s:-\frac{1}{2}\left(p_{0}{ }^{2}+k(s) q_{0}{ }^{2}\right):\right)\left(\gamma(s) q_{0}{ }^{2}+2 \alpha(s) q_{0} p_{0}+\beta(s) p_{0}{ }^{2}\right):\right) \\
& =\exp \left(:-\frac{1}{2} \mu\left(c-d s\left[-\frac{1}{2}\left(p_{0}^{2}+k(s) q_{0}^{2}\right), \gamma(s) q_{0}^{2}+2 \alpha(s) q_{0} p_{0}+\beta(s) p_{0}{ }^{2}\right]\right):\right) \\
& \Rightarrow \frac{\mathrm{d} \alpha}{\mathrm{ds}}=\beta \mathrm{k}-\gamma ; \frac{\mathrm{d} \beta}{\mathrm{ds}}=-2 \alpha ; \frac{\mathrm{d} \gamma}{\mathrm{ds}}=2 \alpha \mathrm{k} .
\end{aligned}
$$

Using the relations of line (64) and the relation $1+\alpha^{2}=\beta \gamma$, we get

$$
\Delta \Phi_{s_{0} s_{1}}=\int_{s_{0}}^{s_{1}} \frac{k(s) d s}{\gamma}=\int_{s_{0}}^{s_{1}} \frac{\gamma+\frac{d \alpha}{d s}}{\beta \gamma} d s=\int_{s_{0}}^{s_{1}} \frac{1}{\beta} d s+\left.\tan ^{-1}(\alpha)\right|_{s=s_{0}} ^{s=s_{1}}
$$

In equation (65), we explicitly demonstrated that matched $\left(\alpha_{1}=\alpha_{2}\right)$ locations are separated by the same phase advance. If we then multiply $\rho^{\prime}-1$ by $\rho$ ( equation (53) and (62) ), we get an another advertised result: 


$$
\left(\begin{array}{cc}
\sqrt{\gamma} & \frac{\alpha}{\sqrt{\gamma}} \\
0 & \frac{1}{\sqrt{\gamma}}
\end{array}\right)\left(\begin{array}{cc}
\sqrt{\beta} & 0 \\
\frac{-\alpha}{\sqrt{\beta}} & \frac{1}{\sqrt{\beta}}
\end{array}\right)=\left(\begin{array}{cc}
\frac{1}{\sqrt{1+\alpha^{2}}} & \frac{\alpha}{\sqrt{1+\alpha^{2}}} \\
\frac{-\alpha}{\sqrt{1+\alpha^{2}}} & \frac{1}{\sqrt{1+\alpha^{2}}}
\end{array}\right) .
$$

As we said, the matrix in (66) is a rotation; two definitions of $\rho$ can only differ by a phase. In fact, the angle of the rotation in (66) is $\tan ^{-1}(\alpha)$, in perfect agreement with (65).

We close the discussion by a remark on Dragt's original definition of the phase advance. In reference 11, Dragt did defined the canonical transformation $\rho$ in terms of maps. However, he artificially introduced the time-like variable $s$ of the original Hamiltonian in the definition of $\rho$. As a result it was not true that

$$
\mathbf{M}_{\mathbf{i}}=\mathbf{M}_{\mathbf{j}} \Rightarrow \rho\left(\boldsymbol{M}_{\mathbf{i}}\right)=\rho\left(\boldsymbol{M}_{\mathbf{j}}\right)
$$

Although it is conceivable to imagine cases were (67) should be discarded on the basis of connecting two different types of perturbations, it is unacceptable to do it at random using the Hamiltonian parameter s. In that sense, Dragt's treatment was not totally Hamiltonian-free. 


\section{Conclusion}

We would like to summarize the actual achievements of the Hamiltonian-free theory. Firstly, as we emphasize throughout this paper, our approach goes directly to the quantities of interest, this simplifies greatly the theory for any representation of the map.

Secondly, our ability to generate and analyse Taylor series maps allows us to study arbitrarily complex systems, in particular, circular accelerators. One can define a Floquet ring and perturbed it by Hamiltonian or/and stochastic effects. For example, one can easily implement the stochastic calculation of the final emmitances proposed by Chao in any tracking code ${ }^{[38]}$. We are no longer restricted to simple models: this could become important in understanding the behavior of small light sources because of the non-trivial fringe fields they generate.

Finally, other areas of physics could benefit from such an approach. For example, in the design of toroidal stellerators, one can show that the magnetic field line pattern is (in some variables) a two dimensional symplectic map. The computation of this map is extremely complex and tedious since one must integrate Biot-Savart law around the stellarator. Hanson and Cary, in a paper on the stochastic nature of this map ${ }^{[39]}$, did exactly that. Had they known of the automatic differentiation of Berz, they could have attempted to compute a one-turn map with some dependence on the current parameters they used to reduce the stochasticity. In fact, the stellarator problem seems to typify a proper use of a map based theory: the map is simple (2-d) but the Hamiltonian 
generating it is extremely complex (i.e. Maxwell's equations). In addition, the field line are best integrated using non-Hamiltonian variables. One can convert the 2-d map into canonical variables at the end of the calculation just before feeding it into some canonical perturbation theory algorithm.

By this example, we just wanted to point out the generality of certain concepts. Since not all problems are identical, we are convinced that the greater the selections of tools, the more efficiently a researcher or designer can attack a complex problem.

\section{Prospect for the Future}

We started this paper by pointing out that the essential problem of accelerator dynamics is to study the long term stability of the one-turn map. In fact, from a strict analysis of error propagation, accelerator simulation integrate the motion of particles far beyond a rigourously reasonable limit. Given this fact, can we then use a one turn map in our simulation ? Experience has shown that truncated Taylor series produced non-symplectic maps with vastly different long term behavior. Indeed, the motion can settle on a fixed point in phase space after a relatively short number of turns despite a highly accurate Taylor series representation. However, with our ability to extract maps and manipulate them, we can re-express the Taylor series representation into various exactly symplectic representations. This is being extensively studied at the moment driven by projects such as third generation synchrotron light sources, small "pocket" light source and large hadron rings like the comtemplated SSC (Superconducting Super Collider). 
We are also trying to understand quasi-symplectic maps. For example, in light sources and so-called "beauty factories", electrons radiate a substantial amount of energy. In the classical regime, this leads to a nonlinear map with damping. This map can be easily extracted with the automatic differentiation techniques but its analysis in the nonlinear régime will require new developments beyond those advertized in this paper. In particular, it will not be possible to express the one turn-maps using symplectic Lie generators, but hopefully a new expanded set of Lie generator can be found.

All these problems and others are now within reach thanks to the type of re-thinking introduced in this paper.

\section{Acknowledgements}

I would like to thank Prof. Alex Dragt who has been my mentor and has initiated this vastly expanded usage of maps in accelerator theory. More recently, I had the pleasure to collaborate with John Irwin of SLAC. Finally, I am extremely grateful to Martin Berz for developing the Differential Algebra Package and attaching to it extra subroutines to ease the development of map normalization algorithms. His work on automatic differentiation re-inforced the seriousness and generality of the map approach to accelerator design. The usefulness of his methods go far beyond the domain of dynamical systems.

Finally, I am also grateful to my co-workers and, in particular, to my supervisor Dr. Swapan Chattopadhyay for providing the necessary support. 
Appendix 1: A map Derivation of the New Hamiltonian using the adjoint representation of the Lie operator Algebra

Assuming that we are interested in all surfaces of sections, the ring becomes an "o-tuple" described equations (2), (8) and (9). In terms of maps, they take the form:

$$
\begin{aligned}
& \frac{d}{d \varepsilon} \mathbf{A}^{-1}\left(z_{0} ; s ; \varepsilon\right)=A^{-1}\left(z_{0} ; s ; \varepsilon\right):-w\left(z_{0} ; s ; \varepsilon\right): \\
& \frac{d}{d s} M\left(z_{0} ; s\right)=M\left(z_{0} ; s\right):-K\left(z_{0} ; s\right): \\
& K\left(z_{0} ; s\right)=\mathbf{H}\left(z_{0} ; s\right)\left(H\left(z_{0} ; s\right)+\int_{0}^{\varepsilon=1} d \varepsilon \boldsymbol{A}^{-1}\left(z_{0} ; s\right) \frac{\partial}{\partial s} w\left(z_{0} ; s ; \varepsilon\right)\right) \\
& M\left(z_{0} ; s=s_{0}\right)=E=\text { identity }
\end{aligned}
$$

Equations (A1) must be integrated from $s_{0}$ to $s_{0}+1$ if one is to obtain a one-turn map. For a complete normalization, it must accompanied by the boundary conditions:

$$
\begin{aligned}
& K\left(z_{0} ; s\right)=\frac{\partial}{\partial s} \Phi_{S}(J) \\
& \mathbf{A}^{-1}\left(z_{0} ; s ; \varepsilon=1\right)=\mathbf{A}^{-1}\left(z_{0} ; s+1 ; \varepsilon=1\right)
\end{aligned}
$$

The periodicity of (A2b) and the normalized form imposed on $\mathrm{K}$ in 
equation (A2a) renders the direct solution of equations (A1) and (A2) very difficult unless the original Hamiltonian $\mathrm{H}$ is simple. The process involves the computation of various Green's functions on which the proper boundary conditions are imposed. (see reference 6.)

In this appendix, we will concentrate on equation (A1c) (first obtained by Dewar ${ }^{[40]}$ and then Cary $\left.{ }^{[41]}\right)$. It can be derived very elegantly using homomorphic Lie algebras. Let us denote by $\mathrm{N}$ the map generated by $\mathrm{H}$ :

$$
\frac{d}{d s} N\left(z_{0} ; s\right)=N\left(z_{0} ; s\right):-H\left(z_{0} ; s\right):
$$

Using Lie properties of maps, we can write $\mathbf{M}\left(\mathbf{z}_{0} ; \mathbf{s}\right)$ as:

$$
M_{s}=A_{0} N_{s} A_{s}{ }^{-1}
$$

In equation (A4) all the maps transform functions of the initial coordinates $\mathbf{z}_{\mathbf{0}}$. First $\boldsymbol{A}_{\mathbf{0}}\left(\mathbf{z}_{\mathbf{0}}\right)$ brings us to the original variables; these are propagated with the help of $\mathbf{N}_{\mathbf{s}}\left(\mathbf{z}_{\mathbf{0}}, \mathbf{s}_{0}\right)$ and finally they are taken back into the new variables by $\boldsymbol{A}_{\mathbf{S}}\left(\mathbf{z}_{\mathbf{0}}\right)^{-1}$. To get the new Hamiltonian $\mathrm{K}$, we take the time derivative of (A4) with respect to s:

$$
\begin{aligned}
& \frac{d}{d s} M_{s}=A_{0}\left(\frac{d}{d s} N_{s}\right) A_{s}{ }^{-1}+A_{0} N_{s}\left(\frac{d}{d s} A_{s}{ }^{-1}\right) \\
& =A_{0} N_{S} A_{S}{ }^{-1} A_{S}:-H: A_{S}{ }^{-1}+A_{0} N_{S} A_{S}{ }^{-1} f_{S}\left(\frac{d}{d s} A_{S}{ }^{-1}\right)
\end{aligned}
$$


$=M_{s}\left(:-A_{s} H:+A_{s}\left(\frac{d}{d s} A_{s}^{-1}\right)\right)$

Comparing (A5) with (A1b), we conclude that $\mathbf{A}_{\mathbf{s}}\left(\frac{\mathrm{d}}{\mathrm{ds}} \mathbf{A}^{-1}\right)$ must be a Lie operator.

To evaluate it, we use the equation of $\mathbf{A}_{\mathbf{S}}$ and $\mathbf{A}_{\mathbf{s}}{ }^{-1}$ :

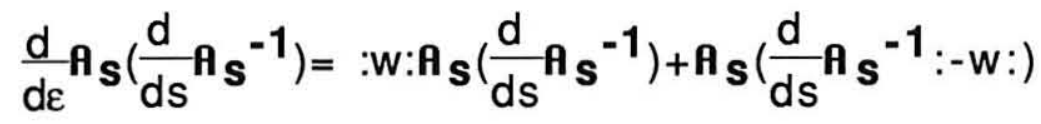

$$
\begin{aligned}
& =\left\{: w:, \mathbf{A}_{\mathbf{s}}\left(\frac{d}{d s} \mathbf{A}^{-1}\right)\right\}+:-\frac{\partial}{\partial s} w: \quad ; \quad \frac{\partial}{\partial s} w=\frac{d}{d s} w .
\end{aligned}
$$

Here,$\{$,$\} denotes the commutator of two Lie operators. Denoting by \mathbf{G}$ the operator $\boldsymbol{A}_{\mathbf{s}}\left(\frac{\mathrm{d}}{\mathrm{ds}} \mathbf{A}_{\mathbf{s}} \mathbf{- 1}^{\mathbf{1}}\right)$, we rewrite (A6) :

$$
\frac{d}{d \varepsilon} \mathbf{G}-\# \mathbf{w} \# \mathbf{G}=:-\frac{\partial}{\partial s} \mathbf{w}:
$$

Here, \# w \# is a super operator which acts on the space of Lie transforms by taking a commutator. These commutators form a Lie algebra. Notice the homomorphisms between the Lie algebra of super operators, Lie operators and Poisson brackets ${ }^{[42]}$ :

$$
\# f: g:=\{: f:, g:\}=:[f, g]:
$$


To solve (A7), we make use of (A8) by writing $\mathbf{G}$ in terms of an $\varepsilon$ dependent super operator.

$$
\mathbf{G}(\varepsilon)=\mathrm{P}_{\mathbf{G}_{0}}
$$

We first solve the homogeneous equation:

$$
\frac{d}{d \varepsilon} \mathrm{P}-\# w \# \mathrm{P}=0 \Rightarrow \mathrm{P}=\boldsymbol{\theta}(\# \mathrm{w}(\varepsilon) \#)
$$

To get $\mathrm{P}$ in (A10), we noticed that the formal functional dependence of

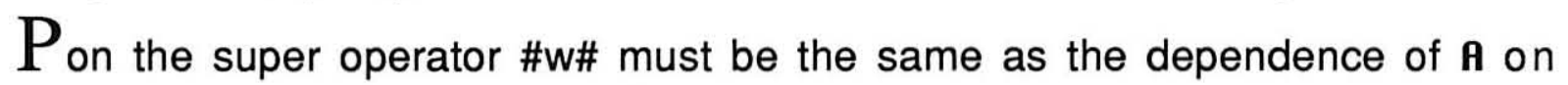
Lie operator :w: similarly $\mathrm{P}^{-1}$ must have the functional dependence on \#-w\# as $\mathbf{A}^{-1}$ has on :-w:.

To solve the non-homogeneous equation, we allow $\mathbf{G}_{\mathbf{0}}$ to depend on $\varepsilon$ (variation of parameters). We obtain for the particular solution $\mathbf{G}_{\mathbf{p}}$ :

$$
\mathbf{G}_{\mathbf{p}}=\mathbf{A}(\# \mathbf{w}(\varepsilon) \#) \int_{0}^{\varepsilon} \mathrm{d}^{\prime} \mathbf{H}^{-1}\left(\#-\mathbf{w}\left(\varepsilon^{\prime}\right) \#\right):-\frac{\partial}{\partial s} \mathbf{w}\left(\varepsilon^{\prime}\right):
$$

The general solution is the sum of the homogeneous and particular solution:

$$
\mathbf{G}=\mathbf{A}(\# \mathrm{w}(\varepsilon) \#)\left(\mathbf{G}_{\mathbf{0}} \mathbf{0}_{0}^{\varepsilon} \mathrm{d} \varepsilon^{\prime} \mathbf{H}^{-1}\left(\#-\mathrm{w}\left(\varepsilon^{\prime}\right) \#\right):-\frac{\partial}{\partial \mathrm{s}} \mathrm{w}\left(\varepsilon^{\prime}\right):\right)
$$

We impose the boundary condition at $\varepsilon=0$ : 


$$
\begin{aligned}
(\mathbf{G}(\varepsilon=0) & \left.=\mathbf{0} \Rightarrow \mathbf{G}_{\mathbf{0}}=\mathbf{0}\right) \\
\therefore \text { hence } \mathbf{G} & =\mathbf{H}(\# \mathbf{w}(\varepsilon) \#) \int_{0}^{\varepsilon} \mathrm{d} \varepsilon^{\prime} \boldsymbol{H}^{-1}\left(\#-\mathbf{w}\left(\varepsilon^{\prime}\right) \#\right):-\frac{\partial}{\partial \mathrm{s}} \mathbf{W}\left(\varepsilon^{\prime}\right):
\end{aligned}
$$

Finally here enter the homomorphisms between the three Lie algebras of line (A8) :

$$
\begin{aligned}
& \boldsymbol{H}(\# \mathbf{w}(\varepsilon) \#) \int_{0}^{\varepsilon} d \varepsilon^{\prime} \boldsymbol{H}^{-1}\left(\#-w\left(\varepsilon^{\prime}\right) \#\right):-\frac{\partial}{\partial s} w\left(\varepsilon^{\prime}\right): \\
& =:-\boldsymbol{H}(: w(\varepsilon):) \int_{0}^{\varepsilon} d \varepsilon^{\prime} \boldsymbol{H}^{-1}\left(:-w\left(\varepsilon^{\prime}\right):\right) \frac{\partial}{\partial s} w\left(\varepsilon^{\prime}\right):
\end{aligned}
$$

Substitution of (A14) into (A5) gives the advertized result. 


\section{Appendix 2: The Uniqueness of $\mathbf{R}$ and the Phase Advance}

We first prove that given a map $M$, the assumed analyticity of the similarity transformation insures the uniqueness of $\mathbf{R}$.

We start by postulating the existence of two normalized rotations:

$$
H_{1} M H_{1}^{-1}=R_{1} \text { and } H_{2} M H_{2}^{-1}=R_{2} \text {; }
$$

which in turns implies that

$$
\mathrm{A}_{2} \mathrm{H}_{1}^{-1} \mathrm{R}_{1} \boldsymbol{H}_{1} \mathrm{H}_{2}^{-1}=\mathrm{R}_{2}
$$

This is a generalization of equation (33) for the phase advance:

$$
\exp \left(: \boldsymbol{A}_{2} \mathbf{A}_{\mathbf{1}}{ }^{-\mathbf{1}}\left(-\mu_{1} \cdot \mathbf{J}+D_{1}(\mathbf{J})\right):\right)=\exp \left(:-\mu_{2} \cdot \mathbf{J}+D_{2}(\mathbf{J}):\right)
$$

Using analyticity, we follow Dragt and Finn ${ }^{[43]}$ by factorizing $\mathbf{A}_{2} \mathbf{A}_{\mathbf{1}}{ }^{-\mathbf{1}}$ :

$$
\mathbf{A}_{2} \mathbf{A}_{1}^{-1}=\ldots \mathbf{r}_{k} r_{k-1} \ldots r_{1} r_{L} \text {; }
$$

where... $\mathbf{r}_{\mathrm{k}}=\exp \left(: \mathrm{p}_{\mathrm{k}+2}:\right)$ and $\mathrm{p}_{\mathrm{k}+2}$ is a homogeneous polynomial of degree $k+2$ in the phase space variables.

Except for a mere relabelling of the planes, let us assume that the uniqueness of $\mathbf{R}$ is true in the linear regime (the proof would be quite different for linear maps and it amounts to the uniqueness of eigenvalues!). Then (B3) takes the form: 


$$
\exp \left(: . . \mathbf{r}_{k} \mathbf{r}_{\mathrm{k}-1} \ldots \mathbf{r}_{1}\left(-\mu \cdot \mathbf{J}+D_{1}(\mathbf{J})\right):\right)=\exp \left(:-\mu \cdot \mathbf{J}+D_{2}(\mathbf{J}):\right)
$$

To go further, we proceed by induction. Assuming that for $k<j-1$, the $r_{k}$ 's are rotations, we collect the terms of order $j+2$ and get an equation for $p_{j+2}$ :

$$
: \mu \cdot \mathbf{J}: p_{j+2}=D_{2}(J)-\left.D_{1}(J)\right|_{j+2} \text { component }
$$

Using the direct sum decomposition (or the eigenbasis) and the mutual irrationality of the tunes (line 22,23 , and 25 ), we conclude from (B6) that $\mathrm{p}_{\mathrm{j}+2}$ cannot contain anything from $\operatorname{Im}(: \mu \cdot \mathrm{J}:)$. In addition, since the left hand side of (B6) must be in $\operatorname{Ker}(: \mu \cdot \mathrm{J}:)$, the only consistent solution to (B6) is :

$$
\mathrm{p}_{\mathrm{j}+2} \in \operatorname{Ker}(: \mu \cdot \mathbf{J}:) \text { and } D_{2}(\mathbf{J})-\left.D_{1}(\mathbf{J})\right|_{j+2 \text { component }}=0 \text { QED. }
$$

The rest follows by induction, starting with $j=1$.

This also proves the statement on the phase advance because it is a special case of (B1).

Finally, by generalizing equation (14) to the nonlinear maps, we can easily that $\mathbf{R}$ does not depend on the location: 


$$
\begin{aligned}
& M_{j}=N_{i j}{ }^{-1} M_{i} N_{i j} \text { where } N_{i j}=\prod_{k=i}^{j-1} N_{k} k+1 \\
& \boldsymbol{A}_{\mathbf{i}} \mathbf{M}_{\mathbf{i}} \boldsymbol{A}_{\mathbf{i}}^{-1}=R_{\mathbf{i}} \text { and } \boldsymbol{A}_{\mathbf{j}} \mathbf{M}_{\mathbf{j}} \boldsymbol{\theta}_{\mathbf{j}}^{-1}=R_{\mathbf{j}} \\
& (\mathrm{B} 8 \mathrm{a}) \text { and }(\mathrm{B} 8 \mathrm{~b}) \Rightarrow \boldsymbol{A}_{\mathbf{j}} \mathbf{N}_{\mathbf{i j}}{ }^{-1} \mathbf{M}_{\mathbf{i}} \mathbf{N}_{\mathbf{i j}} \mathbf{H}_{\mathbf{j}}^{-1}=\mathbf{R}_{\mathbf{j}}
\end{aligned}
$$

Lines (B8a) and (B8b) violate the uniqueness of $\mathbf{R}$ for a given map $\mathbf{M}$. 


\section{REFERENCES}

1. E.D. Courant, and H.S. Snyder, Annals for Physics 3, 1 (1958).

2.H. Meier and K.R. Symon, Analytical and Computational Studies on the Interaction of a Sum and a Difference Resonance, Proceedings of the International Conference on HighEnergy Accelerators and Instrumentation, Cern, ed. L. Kowarski, Cern. Publ., (1959). See also: .L. J. Laslett, E.M. MacMillan and J. Moser, Long Term Stability for Particle Orbits, Courant Institute of Mathematical Science, New York University, NYO-1480-101, (1968). Also in this reference, one finds some comments by Moser on the Birkhoff normalization of the Hamiltonian.

3. The list of kick code is endless. For example, R. Talman and L.

Schachinger, Teapot: A Thin Element Accelerator Program for Optics and Tracking,Part. Accel.,22, (1987). or A. Wrulich, Racetrack, A Computer Code for the Simulation of Nonlinear Motion in an Accelerator, DESY 84026 (1984).

4. A. Schoch, Theory of Linear and Nonlinear Perturbations of Betatron Oscillations in Alternating Gradient Synchrotrons, CERN 57-21, (1958).

5.See ref. 2.

6. L. Michelotti, Moser-like Transformations using the Lie Transform, Part. Accel.,16, (1985).

7. A. Chao, Evaluation of Beam Distribution Parameters in an Electron 
Storage Ring, J. Appl. Phys., 50, (1979).

8. In single pass systems, such as spectrometers, the extraction of Taylor series maps is a time honored practice. Indeed the aberrations of interest are directly the coefficients of the Taylor series. Again the list of power series codes is endless.

9. A.J. Dragt and John M. Finn, Normal Form for Mirror Machine Hamiltonians, J. Math. Phys.,20,(1979).

10. A.J. Dragt and John M. Finn, Insolubility of Trapped Particle Motion in a Magnetic Dipole Field, J. Geo. Phys.,81,13,(1976).

11. See Ref. 9.

12. A.J. Dragt,et al., Lie Algebraic Treatment of Linear and Nonlinear Beam Dynamics, Ann. Rev. Nucl. Sci.,38, (1988).

13. A.J.Dragt, Nonlinear Lattice Functions,Proc. 1984 Summer Study on the Design and Utilization of the Superconducting Super Collider, Snowmass, Colorado, ed. R. Donaldson, J. Morfin. New York: Div. Part. Fields, Am. Phys. Soc. (1985).

14. E. Forest, Lie Algebraic Maps and Invariants Produced by Tracking Codes, Part. Accel. 22, (1987).

15. M. Berz, Differential Algebraic Description of Beam Dynamics to Very High Orders, Part. Accel., 24, (1989).

16. E. Forest, J. Irwin and M. Berz, Normal Form Methods for Complicated 
Periodic Systems: A Complete Solution Using Differential Algebra and Lie Operators, Part. Accel., 24, (1989).

17. A.J. Dragt, Lectures on Nonlinear Orbit Dynamics, Am. Inst. Phys. Conf. Proc. No. 87, ed. R.A. Carrigan et al.New York AIP (1982).

18. A.J. Dragt and John M. Finn, Lie Series and Invariant Functions for Analytic Symplectic Maps, J. Math. Phys.,17, (1976).

19.A.J. Dragt and E. Forest, Computation of Nonlinear Behavior of Hamiltonian Systems Using Lie Algebraic Methods, J. Math. Phys.,24 (1983).

20. See ref. 19.

21. R. L. Dewar, Renormalized Canonical Perturbation Theory for Stochastic Propagators, J. Phys. A9, (1976).

22. J. Cary, Lie Transform Perturbation Theory for Hamiltonian Systems, Physics Reports (review section of Physics Letters) 79 (1981).

23. R.D. Ruth, A Canonical Integration Technique, IEEE Trans. Nucl. Sci., NS-30 (1983).

In addition, R. Ruth and the author are preparing a paper on symplectic integration and its connection with the Lie representation of maps near the identity (Accepted in Physica D, 1990) Furthermore, the author has derived a $6^{\text {th }}$ order integrator using the Lie methods (see LBL-Report 28684 and also submitted to the Journal of Computational Physics) 
24. Because we use symplectic integrators, the maps we extract are Hamiltonian in nature to computer accuracy. The theory presented here still applies for non-symplectic integrators. Obviously, the violation of 'symplecticity' is of the same order as the error due to the finite size of the integration steps.

25. See ref.15.

26. See ref. 16.

27. R.L.Warnock, R.D. Ruth, W. Gabella and K. Ecklund, Methods of Stability Analysis in Nonlinear Mechanics, SLAC-PUB-4846, (1989).

28. See refs. 15 and 16.

29. The normalization of a one-turn Taylor series map using the Differential Algebra Package of Berz is explained in details in reference 16. Here we summarize the results de-emphasizing the Taylor series representation. One must realize that even in other representations, normalization procedures are formal manipulations. Most problems are not integrable and therefore the expressions for the invariants are asymptotic series in some smallness parameter.

30. See ref. 18.

31. R. Cushman, A. Deprit and R. Mosak, Normal Form and Representation Theory.,J. Math. Phys.,24, (1983). 
32.See ref. 9.

33.See ref. 9.

34. See ref. 17.

35. See refs. 1 anf 17.

36. See ref 1.

37. D.A. Edwards and L.C. Teng, Parametrization of Linear Coupled Motion in Periodic Systems, IEEE Trans. Nucl. Sci.,NS-20 (1983).

38. See ref. 7.

39. J.D. Hanson and J.R. Cary, Elimination of Stochasticity in Stellarators, Phys. Fluids,27, (1984).

40. See ref. 21 .

41. See ref. 22.

42. See ref. 17.

43. See ref. 18. 


\section{Figure Captions}

1. Schematic view of the Ring $\Re$

2. Schematic view of the second order normalization process. 


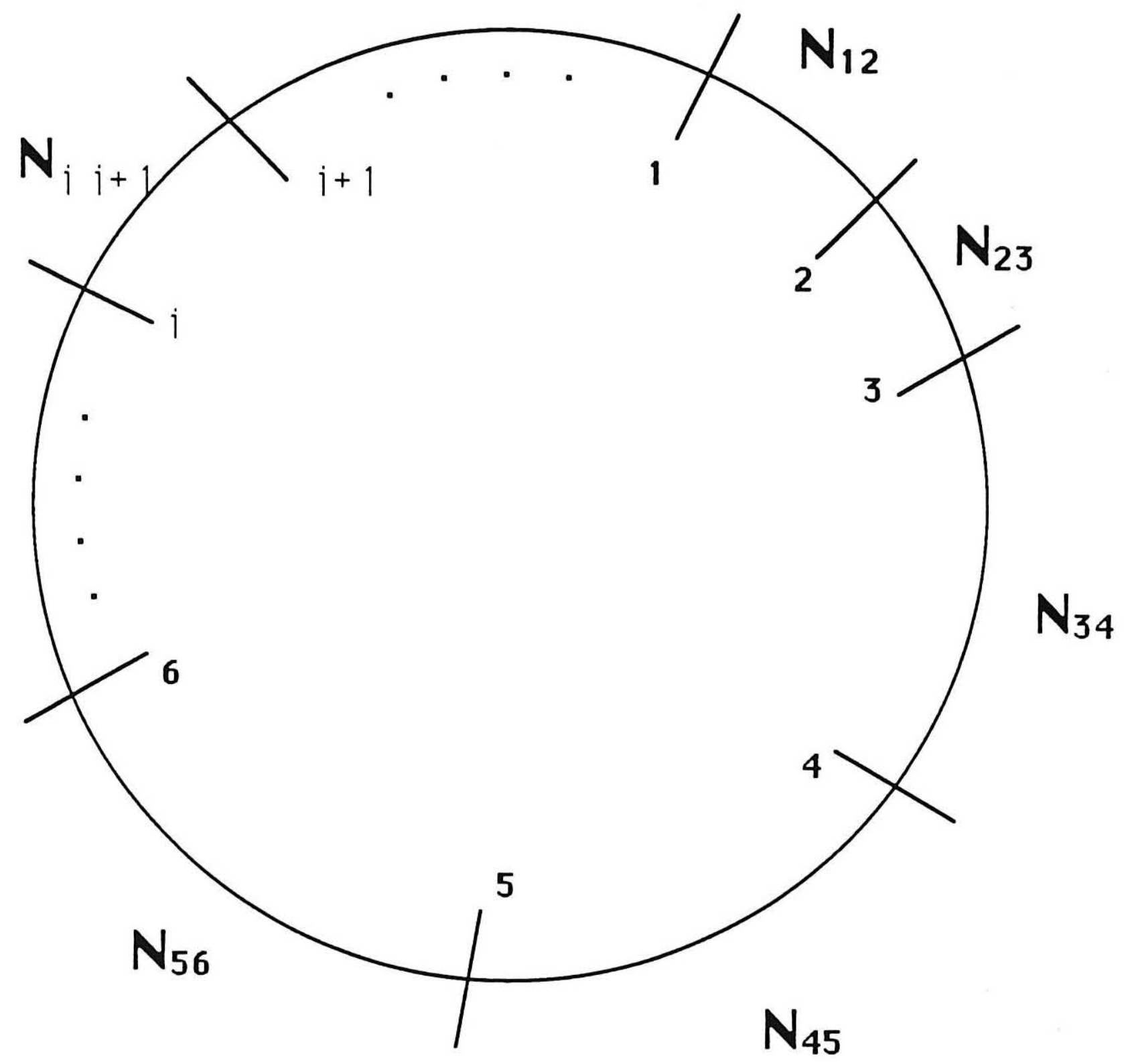

Fig 1. Schematic view of the Ring $\mathfrak{R}$ 


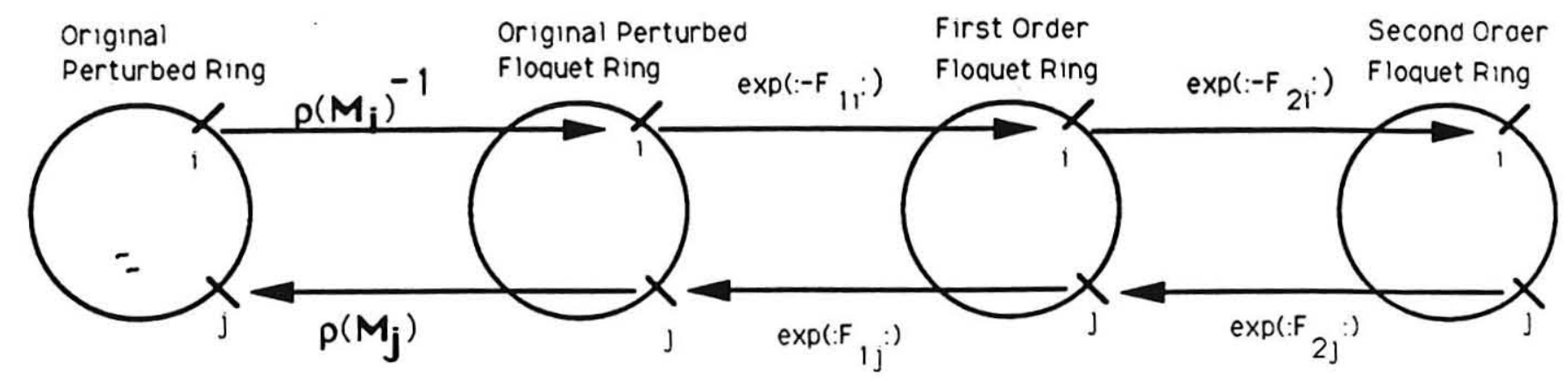

Fig.2: Schematic Process of Second Order Perturbation Theory 